\title{
The Effects of Aging on Indices of Oxidative Stress and Apoptosis in the Female Fischer 344/Nnia X Brown Norway/BiNia Rat Heart
}

\author{
Jacqueline Fannin ${ }^{1,4, \wedge}$, Kevin M. Rice ${ }^{3,4,}$, , Srininvas Thulluri ${ }^{4}$, Ravi Kumar Arvapalli ${ }^{4}$, Pau- \\ lette Wehner ${ }^{2}$ and Eric R. Blough ${ }^{1,4,5, *}$ \\ ${ }^{I}$ Department of Pharmacology, Physiology, and Toxicology, Joan C. Edwards School of Medicine, Marshall University, \\ USA \\ ${ }^{2}$ Department of Cardiology, Joan C. Edwards School of Medicine, Marshall University, Huntington, WV, USA \\ ${ }^{3}$ Department of Internal Medicine, Joan C. Edwards School of Medicine, Marshall University, Huntington WV, USA \\ ${ }^{4}$ Center for Diagnostic Nanosystems, Marshall University, Huntington WV, USA \\ ${ }^{5}$ Department of Pharmaceutical Science Research, School of Pharmacy, Marshall University, Huntington WV, USA
}

\begin{abstract}
Oxidative-nitrosative stress may play a role in age-associated cardiovascular disease as implied by recent studies.However, limited research has been conducted using aged female rodent models. In this study, we examined hearts obtained from 6-, 26-, and 30-month old female Fischer 344/Nnia x Brown Norway/BiNia (F344xBN) rats in order to examine how aging affects levels of cardiac oxidative-nitrosative stress and apoptosis. Oxidative (superoxide anion and 4$\mathrm{HNE}$ ) and nitrosative (protein nitrosylation) stress markers were increased $180 \pm 17 \%, 110 \pm 3 \%$, and $14 \pm 2 \%$, respectively in 30-month hearts compared to the hearts of 6-month female rats. Coincident with these changes in oxidativenitrosative stress, aging was also found to be associated with increases in the number of Tdt-mediated dUTP nick labeling (TUNEL)-positive cardiomyocytes, alterations in the Bax/Bcl-2 ratio, and elevated cleavage of caspase-3. Regression analysis demonstrates significant correlation in the age-associated changes markers of oxidative-nitrosative stress with changes in apoptotic signaling. The findings from this descriptive study imply that age-associated increases in mitochondrial-mediated apoptosis may be associated with the increase in oxidative-nitrosative stress in the aging F344xBN female heart.
\end{abstract}

Keywords: Heart, Aging, Female, ROS.

\section{INTRODUCTION}

Aging is thought to be a major risk factor for cardiovascular disease (CVD), and is associated with a loss of cardiomyocytes by apoptosis, and increases in tissue fibrosis which can lead to systolic and diastolic dysfunction [1-5]. The effect of sex on cardiovascular risk has not been fully elucidated; however, data have suggested that pre-menopausal women have a decreased risk in CVD when compared to men of similar age $[6,7]$. This cardio-protective benefit appears to be lost over time as the risk of developing cardiovascular disease in post-menopausal women appears to be similar to that observed in men of a similar age [8]. The reason(s) for the increased risk of developing CVD following menopause is unclear but may be related to changes in estrogen levels. Recent work has shown that pre-menopausal

*Address correspondence to this author at the Center for Diagnostic Nanosystems, BBSC 241R, Department of Pharmaceutical Science Research, 1700 3rd Ave.Department of Biological Sciences, 1 John Marshall Drive, Marshall University, Huntington, WV 25755-1090;

Fax: 304-692-3267; E-mail: blough@marshall.edu

${ }^{\wedge}$ These authors equally contributed to this article. females appear to exhibit reduced levels of reactive oxygen species (ROS) [9]. Similarly, levels of oxidative-nitrosative stress are reduced in pre-menopausal or estrogen treated rats due to increases in either antioxidant enzyme activity or increases in potential ROS scavenging [10]. Other studies have demonstrated that the mitochondria-mediated pathway of apoptosis was activated in the heart in ovariectomized rats and this appeared to occur coincident with decreased antioxidant enzyme activity and increased indices of oxidative stress $[11,12]$. Although these data are suggestive of estrogen playing a protective role in the cardiovascular system, how the natural loss of estrogen with aging affects cardiac ROS levels is not well known.

Aging in the male Fischer 344/Nnia x Brown Norway/BiNia $(\mathrm{F} 344 \mathrm{xBN})$ rat has been found to be associated with declines in cardiac function, increased markers of oxidative-nitrosative stress, and increased cardiomyocyte apoptosis [13]. Whether these changes are also present in the aging female F344xBN heart is currently unclear. Herein, we examine the effects of aging on indices of oxidativenitrosative stress and apoptosis in 6-, 26-, and 30-month old female F344xBN rats. Similar to that seen in the aging 
males, we hypothesized that aging in female F344xBN animals would be characterized by increases in oxidativenitrosative stress and cardiomyocyte apoptosis. The data presented in this descriptive report support this notion and are consistent with the possibility that age-related increases in oxidative-nitrosative stress in the female F344xBN heart could be responsible, at least in part, for the increased cardiomyocyte apoptosis observed in the aging heart.

\section{MATERIALS AND METHODS}

\section{Materials}

Protein kinase B (Akt) Akt [\#9272], phosphor-Akt (Ser473) [\#9271], phosphor-Akt(Thr308) [\#9275], Bcl-2 (50E3) [\#2870], caspase-12 [\#2202], caspase-3 [\#9662], cleaved caspase-3 Asp175 [\#9661], HSP27 [\#2442], HSP90 (\#4877), nitrotyrosine [\#9691], GAPDH [\#2118], 3T3 Control Cell Extracts [\#9203], mouse secondary [\#7076], rabbit secondary [\#7074] and biotinylated protein ladder [\#7727] were from Cell Signaling Technology (Beverly, MA). The antibody for HSP70 [\#sc-1060], HeLa whole cell lysate [sc2200], and L6 +IGF Cell Lysate [sc-24127] were purchased from Santa Cruz Biotechnology Inc. (Santa Cruz, CA). Precast $10 \%$ and $15 \%$ PAGEr Gold Precast Gels were from Lonza (Rockland, ME), Precision Plus Protein Dual Color Standards[\#161-0374] were obtained from Bio-Rad (Hercules, CA,) and ECL western blot detection reagent was from Amersham Biosciences (Piscataway, NJ), Restore western blot stripping buffer was purchased from Pierce (Rockford, IL), and the In Situ Cell Death Detection Kit was procured from Roche Applied Science (Mannheim, Germany).All other chemicals were obtained from Sigma (St. Louis, MO).

\section{Animals}

All animal procedures were conducted under the Animal Use Review Board of Marshall University using the criteria outlined by the Association for Assessment and Accreditation of Laboratory Animal Care (AALAC) as proclaimed in the Animal Welfare Act (PL89-544, PL91-979, and PL94279). Adult (6-months of age, $n=9)$, aged (26-months, $n=8$ ), and very aged (30-months, $\mathrm{n}=8$ ) female $\mathrm{F} 344 \mathrm{xBN}$ rats were obtained from the National Institute of Aging (Bethesda, MD). Two rats per cage were housed in AALAC approved vivarium with a $12 \mathrm{~h}: 12$ hlight-dark cycle at $22 \pm 2^{\circ} \mathrm{C}$. Food and water were provided ad libitum (LabDiet 5001, PMI Nutrition International, LLC, Brentwood, MO). Animals were allowed to recover from shipping for at least two weeks before experimentation and monitored daily. Rats were removed from the study if they demonstrated signs of failure to thrive such as precipitous weight loss, disinterest in environment, or unexpected gait alterations.

\section{Heart Collection}

Rats were anesthetized with an intraperitoneal injection of ketamine $(40 \mathrm{mg} / \mathrm{kg})$ and xylazine $(10 \mathrm{mg} / \mathrm{kg})$ and supplemented as necessary for reflexive response. Hearts were removed following midline laparotomy, placed in KrebsRinger bicarbonate buffer $(118 \mathrm{mM} \mathrm{NaCl}, 4.7 \mathrm{mM} \mathrm{KCl}, 2.5$ $\mathrm{mM} \mathrm{CaCl}, 1.2 \mathrm{mM} \mathrm{KH} \mathrm{PO}_{4}, 1.2 \mathrm{mM} \mathrm{Mg} \mathrm{SO}, 24.2 \mathrm{mM}$ $\mathrm{NaHCO}_{3}$, and $10 \mathrm{mM}$ a-D-glucose; $\mathrm{pH}$ 7.4; equilibrated with
$5 \% \mathrm{CO}_{2} / 95 \% \mathrm{O}_{2}$ at $37^{\circ} \mathrm{C}$ ), massaged to remove any remaining blood, and quickly weighed before snap freezing in liquid nitrogen.

\section{Histological and Immunohistochemical Analysis}

Hearts were sectioned $(8 \mu \mathrm{m})$ onto poly-lysine coated slides using an IEC Minotome Cryostat. The oxidative fluorescent dye, hydroethidine, was used to visualize superoxide in situ as previously described [13]. Digitized images were used to determine average pixel intensity of six randomly positioned regions $\left(1000 \mu \mathrm{m}^{2}\right)$ per cross sectionto calculate the intensity of fluorescent ethidium-stained nuclei. Eight images per section were analyzed with $\geq 500$ nuclei per section examined.

Nitrotyrosine immunoreactivity was visualized with immunofluorescence as detailed by the manufacturer.Briefly, sections were washed three times using a phosphate buffered saline with $0.5 \%$ Tween 20 (PBS-T) at $\mathrm{pH}$ 7.5. Sections were incubated in a humidified chamber at $24^{\circ} \mathrm{C}$ for $1 \mathrm{~h}$ in a blocking solution ( $5 \%$ bovine serum albumin) with the nitrotyrosine antibody (1:1000 in PBS-T) and then washed three times with PBS. Immunoreactivity was visualized following additional incubation for $30 \mathrm{~min}$ with FITC labeled antirabbit IgG (1:200) and counterstaining with DAPI (4, 6diamidino-2-phenylindole; $1.5 \mu \mathrm{g} / \mathrm{ml}$ ). Images were recorded using an Olympus fluorescence microscope (Melville, NY) at $20 \mathrm{X}$.

\section{In situ TUNEL Staining}

Cross-sections $(8 \mu \mathrm{m})$ were fixed with $4 \%$ paraformaldehyde, washed with PBS (pH 7.4), and then permeablized using $0.1 \%$ Triton $\mathrm{X}$ and $0.1 \%$ sodium citrate for $2 \mathrm{~min}$ at $4^{\circ} \mathrm{C}$ before TUNEL staining. Sections were counter- stained for dystrophin immunoreactivity to illuminate the muscle membrane as outlined previously [14]. Terminal deoxynucleotidyl transferase (TdT) and fluorescein-dUTP (TUNEL reaction mixture) was added to the sections before incubating the sections in a dark humidified chamber at $37^{\circ} \mathrm{C}$ for 60 min. After rinsing with PBS, sections were mounted and counterstained with DAPI to visualize nuclei. Three randomly selected regions from each cross-section were digitally recorded with a CCD camera (Olympus, Melville, NY) to visualize TUNEL positive nuclei using an Olympus fluorescence microscope (Melville, NY) with a 20X objective. Control experiments performed in parallel using DNase 1 or without TdTwere used to verify specificity of labeling.

\section{Isolation of Protein Isolates}

Heart samples were pulverized using a mortar and pestle in liquid nitrogen to a fine powder and weighed. TPER Lysis Buffer (10 $\mu \mathrm{L} / \mathrm{mg}$ tissue; Pierce, Rockford, IL, USA) containing protease (P8340, $10 \mu \mathrm{L} / \mathrm{mL}$ Sigma-Aldrich, Inc., St. Louis, MO, USA) and phosphatase inhibitors (P5726, 10 $\mu \mathrm{L} / \mathrm{mL}$, Sigma-Aldrich, Inc., St. Louis, MO, USA) was added to each sample and the samples homogenized for $45 \mathrm{~s}$. After incubation on ice (30 min), this procedure was repeated and the samples were then centrifuged at $14,000 \mathrm{xg}$ at $4^{\circ}$ $\mathrm{C}$ for $5 \mathrm{~min}$. After centrifugation, the supernatants were removed and the protein concentration of each sample was determined in duplicate using the Pierce Reagent Assay 
(Pierce, Rockford, IL). Samples were diluted to a concentration of $1.5 \mu \mathrm{g} / \mathrm{mL}$ in SDS-loading buffer and boiled for 5 min. Thirty micrograms of protein from each sample were separated using $10 \%$ or $15 \%$ SDS-PAGE gels.

\section{Immunoblot Analysis}

Proteins were transferred using standard conditions onto Hybond nitrocellulose membranes after electrophoresis [15]. RapidStain (G-Biosciences, St. Louis, MO) was used to verify the transfer of protein, equal protein loading between samples, and for normalization between gels. Before incubation with primary antibody, membranes were blocked using $5 \%$ milk TBST-T for 1 hour, and then incubated with primary antibody overnight $(1: 1000)$. After extensive washing in TBS-T, membranes were incubated with horseradish peroxidase-(HRP)-labeled IgG secondary antibodies for $1 \mathrm{~h}$. Protein bands were visualized with ECL western blotting detection reagent before densitometry usinga flatbed scanner (Epson Perfection 3200 PHOTO) and the AlphaEaseFC imaging software. The integrated optical densities were kept within a linear and non-saturated range by adjusting the exposure time.

\section{Statistical Analysis}

Results are presented as mean \pm SEM. The SigmaStat 11.2 statistical program was used to perform one-way analysis of variance (ANOVA) for overall comparisons as well as to determine group differences using the Student-NewmanKeuls post hoc test where applicable. A $P<0.05$ was accepted as the level of significance.

\section{RESULTS}

Superoxide Production, Markers of ROS Stress, and Protein Nitrosylation are Increased with Aging

Compared to 6-month old animals, body weight and heart weight were significantly higher in the 26- and 30-monthold rat $(\mathrm{P}<0.05)$ (Table 1$)$. Hydroethidine $(\mathrm{HE})$, which emits fluorescence upon oxidation by superoxide to ethidium bromide, was used to semiquantitatively determine superoxide production in heart tissue sections from each age group. Compared to 6-month old animals, ethidium fluorescence was $239 \%$ and $180 \%$ higher in 26- and 30-month female hearts, respectively $(P<0.05$, Fig. 1). In a similar fashion, aging increased tyrosine nitration in the 26- and 30-month old animals $(\mathrm{P}<0.05$, Fig. 2). The modification of proteins

Table 1. Heart and body weights of aging $F 344 \times B N$ female rats

\begin{tabular}{|l|l|l|l|}
\hline \multicolumn{1}{|c|}{ Group } & \multicolumn{1}{|c|}{ Body wt (g) } & \multicolumn{1}{|c|}{ Heart wt (g) } & HW/BW ratio (x100) \\
\hline \hline $\mathbf{6 m}$ & $230.5 \pm 5.3$ & $0.76 \pm 0.03$ & $0.330 \pm 0.009$ \\
\hline $\mathbf{2 6 m}$ & $274.0 \pm 4.9^{*}$ & $0.95 \pm 0.01^{*}$ & $0.334 \pm 0.019$ \\
\hline $\mathbf{3 0 m}$ & $321.3 \pm 7.2^{*} \dagger$ & $1.12 \pm 0.05^{* \dagger}$ & $0.351 \pm 0.011$ \\
\hline
\end{tabular}

An asterisk $(*)$ and a cross $(\dagger)$ indicate significant differences from the adult (6-month) and aged (26-month) values respectively $(\mathrm{p}<0.05), \mathrm{n}=8$ or 9 hearts per age group.

\section{A.}

$6 m$

$26 \mathrm{~m}$

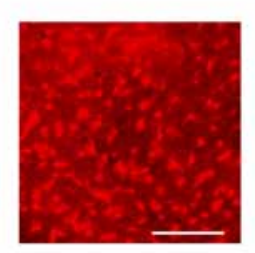

B.

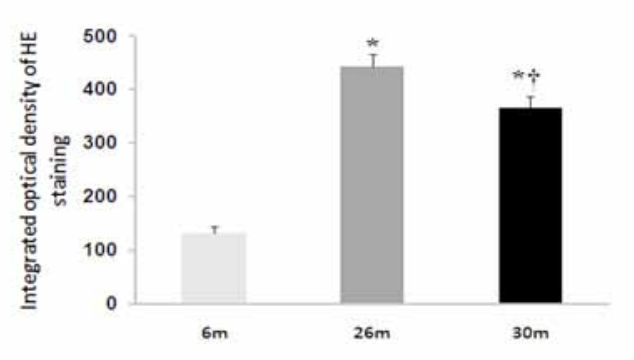

Fig. (1). Aging increases superoxide levels in the female F344xBN heart. A. Superoxide was detected following the formation of dihydroethidium from hydroethidine. Scale bar $=100 \mu \mathrm{m}$. B. Cardiac superoxide levels were quantified by measuring the intensity of ethidiumstained nuclei. * Significantly different from 6-month animals. $\uparrow$ Significantly different from 26 -month animals. 
due to lipid peroxidation was examined using 4-hydroxy-2nonenal (4-HNE), a lipid peroxidation metabolite. Compared to 6-month old animals, 4-HNE levels were $71 \%$ and $110 \%$ higher in the 26- and 30-month hearts $(\mathrm{P}<0.05$, Fig. 3$)$.
Age-associated Cardiomyocyte Apoptosis in the Female F344xBN Heart was Associated with Caspase Activation and Changes in the Bax/Bcl-2 Ratio

Similar to previous work [16-19], TUNEL staining was performed to determine if aging was associated with evi-
A.

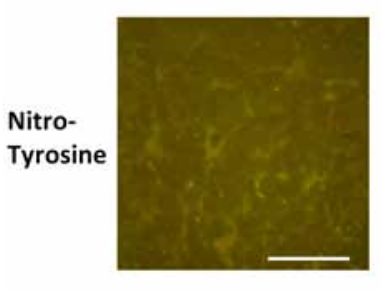

B.

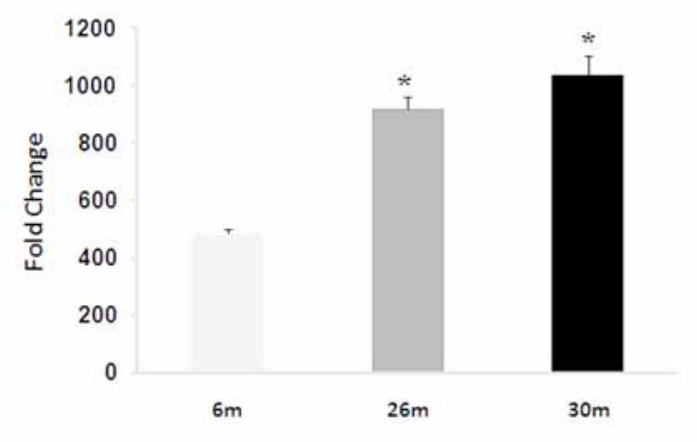

$30 \mathrm{~m}$
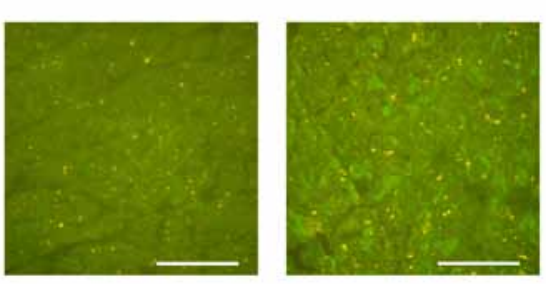

Fig. (2). Aging increases the nitrosylation of tyrosine residues. A. Immunohistochemicalanalysis of tyrosine nitrosylationin the 6-, 26-, and 30 -month female F344xBN heart. Scale bar $=100 \mu \mathrm{m}$. B. Quantification of nitrotyrosine levels.* Significantly different from 6-month animals.
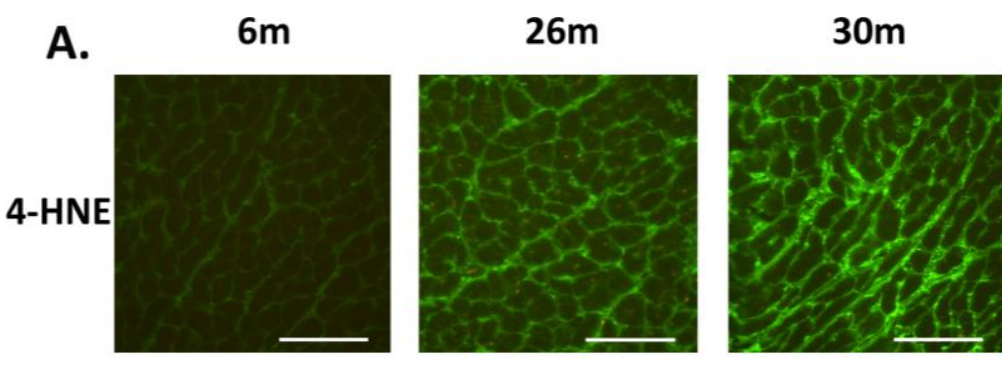

B.

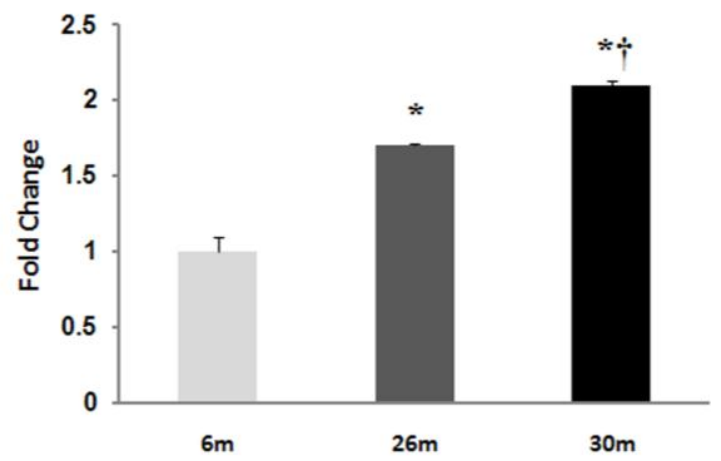

Fig. (3). Lipid peroxidation increases with age in the female F344xBN heart. A. Immunohistochemical analysis of 4-HNE levels in the 6-, 26-, and 30-month female F344xBN heart. Scale bar $=100 \mu \mathrm{m}$. B. Quantification of 4-HNE levels as determined by immunoblotting. * Significantly different from 6 -month animals. $\uparrow$ Significantly different from 26 -month animals. 
dence of increased DNA fragmentation which is suggestive of cellular apoptosis. Compared to that observed in the 6month animals, the number of TUNEL positive nuclei appeared to be increased in 26- and 30-month hearts (Fig. 4). Because caspase- 3 activation is an important step in the initiation of DNA fragmentation / apoptosis, we next examined if aging was associated with increased caspase-3 cleavage. With aging, total caspase-3 expression was $19 \%$ and $29 \%$ lower in the 26-month and 30-month hearts, respectively $(\mathrm{P}<$ 0.05, Fig. 5). Consistent with these data, this decrease in total caspase-3 levels was paralleled by increases in the amount of cleaved caspase-3 (19- and 17-kDa fragments) of $167 \%$ and $290 \%$ at 30 -months $(\mathrm{P}<0.05$; Fig. 5). To confirm these findings we next examined the ratio of Bax (proapoptotic) to Bcl-2 (anti-apoptotic). It is thought that Bax promotes apoptosis by forming a homodimer, which becomes inserted into the mitochondrial membrane where it forms a pore that potentiates the release of cytochrome-C from the mitochondria. With aging, Bax expression was $15 \%$ higher at 30-months when compared to that observed in the 6- and 26-month old animals (Fig. 6). Conversely, the amount of Bcl-2 protein was $13 \%$ lower in the 26-month old animals (Fig. 6). As expected from these data, the Bax/Bcl-2 ratio was $19 \%$ higher in 30-month animals compared to that observed for the 6- and 26-month age groups $(P<0.05)$.

\section{DISCUSSION}

The Fischer 344 x Brown Norway F1 hybrid rat exhibits less age-associated pathologies and an increased longevity in comparison to other rat models [20,21]. Although previous data has demonstrated increased levels of apoptosis and oxidative stress are associated with aging in the male heart, to our knowledge this is the first investigation to examine if these phenomena occur in the naturally menopausal aging female rat model $[13,16]$. Herein, we observed evidence of increased superoxide, protein nitrosylation, and lipid peroxidation with aging (Figs 1-3). Why aging may increase ROS levels is not yet fully understood however recent data has suggested that alterations in enzymatic activity of the xanthine and NADPH oxidoreductases, the mitochondrial electron transport chain, nitric oxide synthase activity, and lipoxygenase/cyclooxygenase may function as potential contributors [22, 23]. In addition ROS production, it is thought that the amount of ROS present in the cell at any one time is determined, at least in part, by the balance between ROS generation and breakdown. The antioxidant enzymes, superoxide dismutase [24], catalase [10], and glutathione peroxidase $(\mathrm{Gpx})$, are thought to remove reactive oxygen species thereby functioning to prevent excessive accumulation of ROS [25]. Recent work has found that antioxidant activity has been shown to be increased and decreased with age in

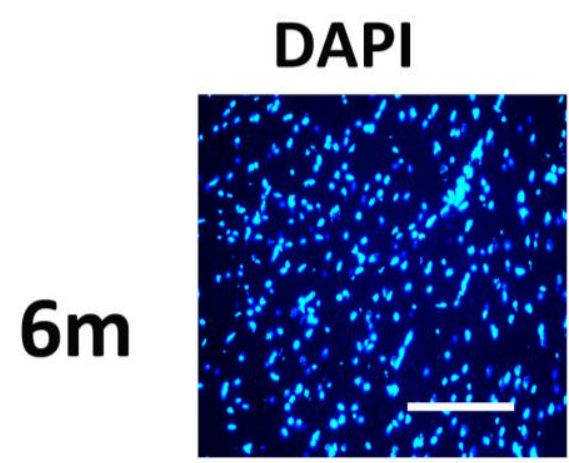

\section{TUNEL}
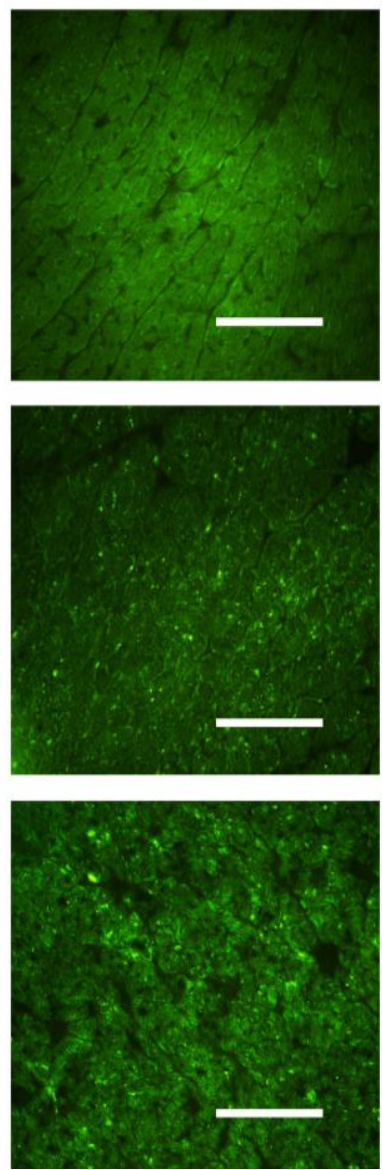

Dystrophin
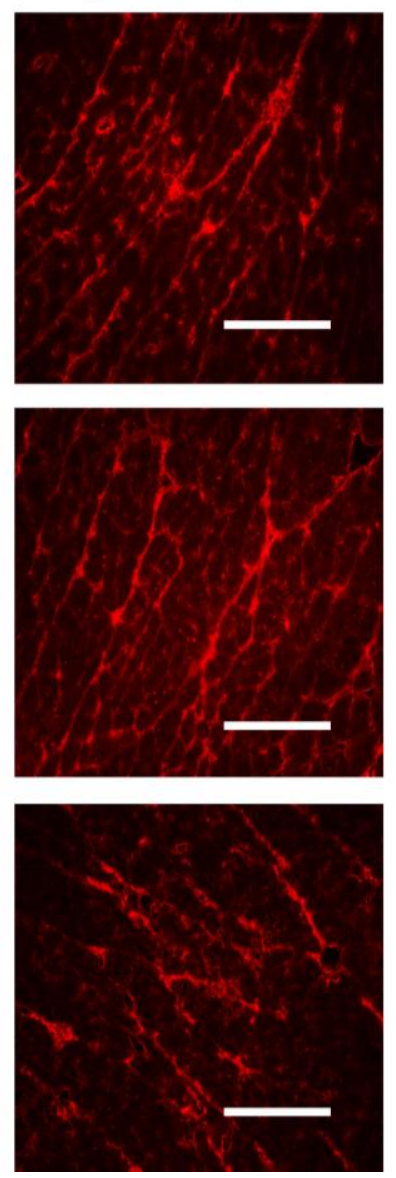

Merged
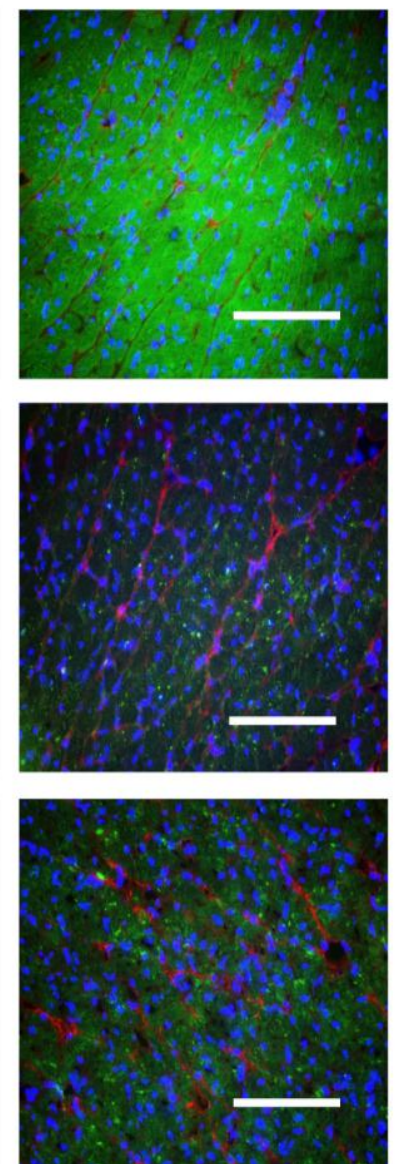

Fig. (4). Indices of apoptosis increase in the aging female heart. Cardiac tissue sections were treated with 4', 6-diamidino-2-phenyllindole (DAPI) and subjected to TUNEL staining to assess DNA fragmentation. Dystrophinimmunohistochemistry was performed to visualize cardiomyocyte borders. Scale bar $=100 \mu \mathrm{m}$. 


\section{Caspase-3}
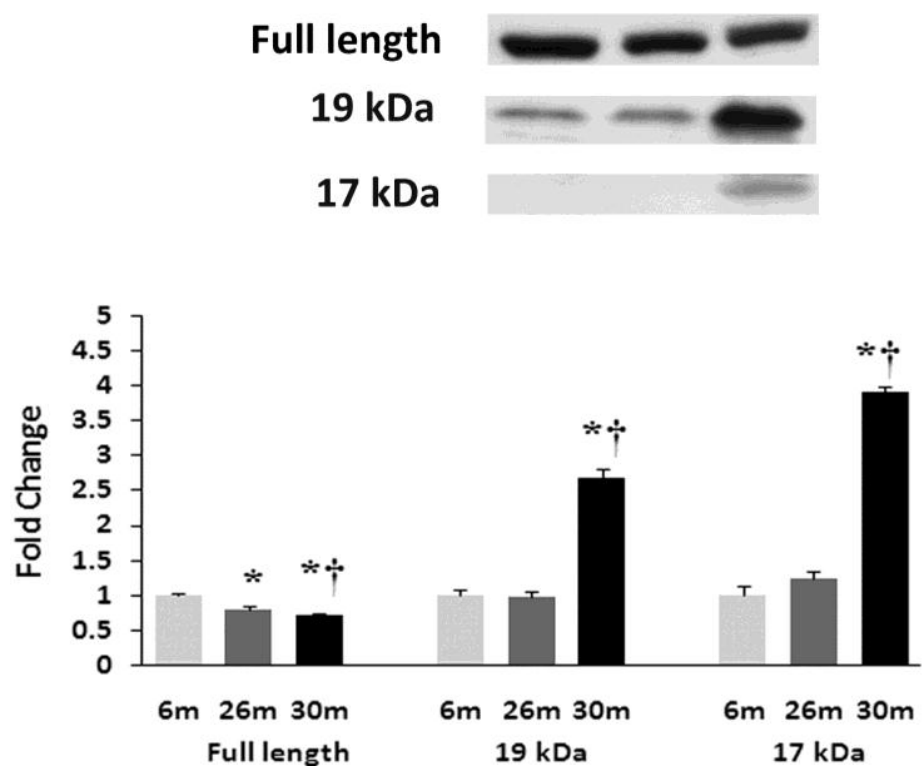

Fig. (5). Aging alters the expression and activation of caspase-3. Expression of caspase-3 in heart tissue of female F344xBN rats as determined by immunoblot analysis. * Significantly different from 6-month animals. $†$ Significantly different from 26 -month animals.

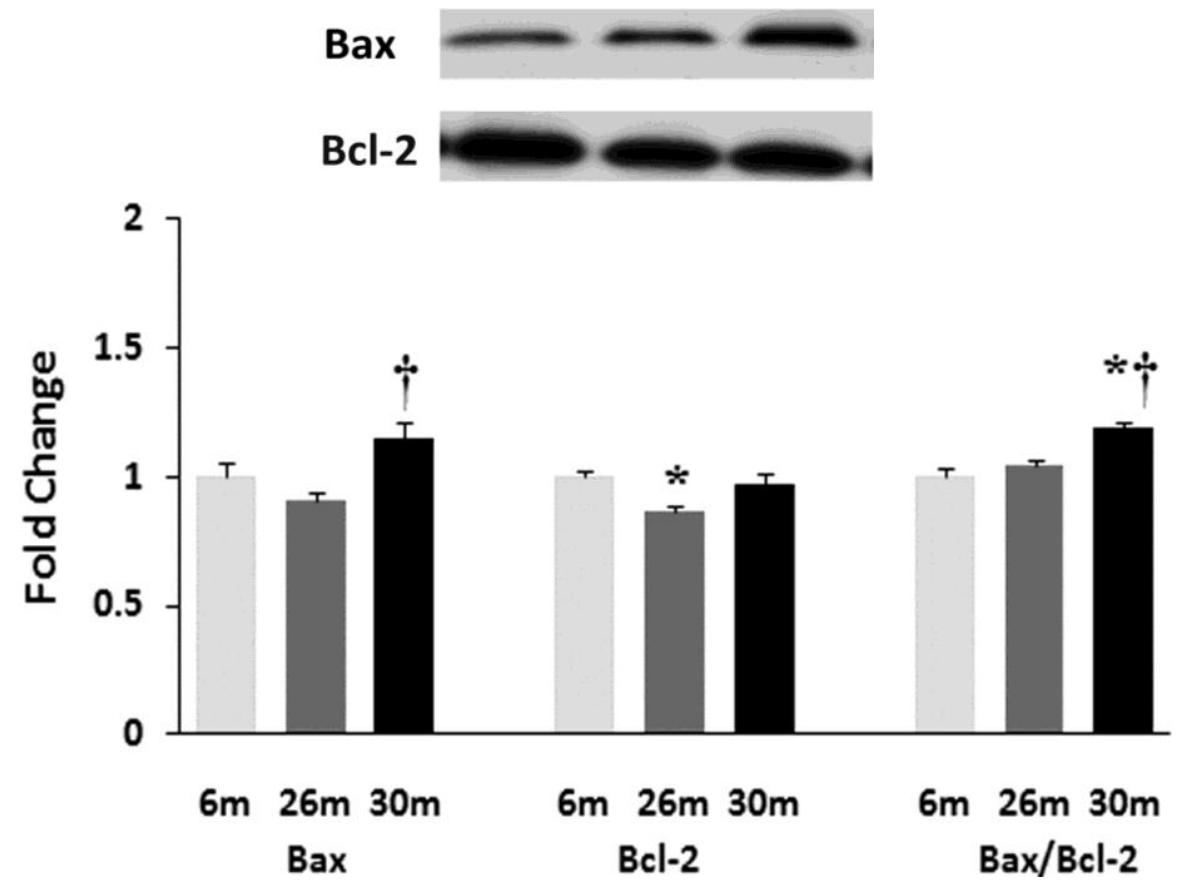

Fig. (6). Aging affects the expression and/or regulation of apoptotic regulators Bax and Bcl-2. Expression of Bax and Bcl-2 protein in female F344xBN rat hearts with age as measured by immunoblot analysis.* Significantly different from 6-month animals. $\uparrow$ Significantly different from 26-month animals.

the rodent heart [26, 27]. Adding to the confusion, we did not find any age-associated alterations in the mRNA expression of SOD1, SOD2, Cat, and Gpx in the female F344xBN heart (data not shown). Whether age-associated dysregulation in the amount of ROS produced, decreases in the abundance of antioxidant enzyme expression, or a combination of the two are responsible for our findings of elevated indicators of ROS in the aging rat heart is currently not known. Future studies designed to directly examine the regulation of some, or several of the ROS generating and buffering pathways, will be required to extend our understanding of the relationship between aging and cardiac ROS regulation.

Elevated levels of superoxide are oftentimes associated with increased nitrosative stress given that superoxide can react with nitric oxide to form peroxynitrite. Peroxynitrite, in turn, can cause tyrosine nitration and the measurement of nitrosylated tyrosine residues is thought to be an established 
Table 2. Regression analysis of expression levels of specific proteins and age, HE staining intensity, protein nitrotyrosine levels, 4HNE levels, heart weight, and TUNEL staining intensity of 6-, 26-, and 30-month female F344xBN hearts

\begin{tabular}{|c|c|c|c|c|c|}
\hline & Age & Heart Weight & HE & Nitro-Tyrosine & 4-HNE \\
\hline \multicolumn{6}{|c|}{ Independent Variable } \\
\hline Age & N.T. & $\begin{array}{l}0.899 * * * \\
(<0.001)\end{array}$ & $\begin{array}{l}0.965^{* * * *} \\
(<0.001)\end{array}$ & $\begin{array}{c}0.849 * * * \\
(0.004)\end{array}$ & $\begin{array}{l}0.969^{* * *} \\
(<0.001)\end{array}$ \\
\hline $\mathrm{HE}$ & $\begin{array}{l}0.965^{* * * *} \\
(<0.001)\end{array}$ & $\begin{array}{c}0.802 * * \\
(0.009)\end{array}$ & N.T. & $\begin{array}{l}0.767 * \\
(0.016)\end{array}$ & $\begin{array}{l}0.905^{* * *} \\
(<0.001)\end{array}$ \\
\hline Heart Weight & $\begin{array}{c}0.286 \\
(0.455)\end{array}$ & N.T. & $\begin{array}{l}0.802 * * \\
(0.009)\end{array}$ & $\begin{array}{l}0.911 * * * \\
(<0.001)\end{array}$ & $\begin{array}{c}0.448 \\
(0.226)\end{array}$ \\
\hline TUNEL & $\begin{array}{l}0.690 \\
(0.40)\end{array}$ & $\begin{array}{c}0.478 \\
(0.193)\end{array}$ & $\begin{array}{l}0.722 * \\
(0.028)\end{array}$ & $\begin{array}{c}0.597 \\
(0.090)\end{array}$ & $\begin{array}{l}0.718^{*} \\
(0.029)\end{array}$ \\
\hline OxyBlot & $\begin{array}{l}0.672 * \\
(0.047)\end{array}$ & $\begin{array}{c}0.607 \\
(0.083)\end{array}$ & $\begin{array}{l}0.688^{*} \\
(0.041)\end{array}$ & $\begin{array}{c}0.483 \\
(0.188)\end{array}$ & $\begin{array}{l}0.682 * \\
(0.043)\end{array}$ \\
\hline Nitro-Tyrosine & $\begin{array}{l}0.849 * * \\
(0.004)\end{array}$ & $\begin{array}{l}0.911 * * * \\
(<0.001)\end{array}$ & $\begin{array}{l}0.767 * \\
(0.016)\end{array}$ & N.T. & $\begin{array}{l}0.907 * * * \\
(<0.001)\end{array}$ \\
\hline 4-HNE & $\begin{array}{l}0.969 * * * \\
(<0.001)\end{array}$ & $\begin{array}{l}0.924 * * * \\
(<0.001)\end{array}$ & $\begin{array}{l}0.905^{* * *} \\
(<0.001)\end{array}$ & $\begin{array}{l}0.907 * * * \\
(<0.001)\end{array}$ & N.T. \\
\hline \multicolumn{6}{|c|}{ Apoptotic Signaling } \\
\hline $\operatorname{Bax}$ & $\begin{array}{c}0.263 \\
(0.493)\end{array}$ & $\begin{array}{c}0.331 \\
(0.385)\end{array}$ & $\begin{array}{c}0.111 \\
(0.835)\end{array}$ & $\begin{array}{c}0.738 \\
(0.094)\end{array}$ & $\begin{array}{c}0.478 \\
(0.338)\end{array}$ \\
\hline $\mathrm{Bcl}-2$ & $\begin{array}{c}0.473 \\
(0.199)\end{array}$ & $\begin{array}{c}0.356 \\
(0.346)\end{array}$ & $\begin{array}{l}0.862 * \\
(0.027)\end{array}$ & $\begin{array}{c}0.394 \\
(0.439)\end{array}$ & $\begin{array}{c}0.637 \\
(0.174)\end{array}$ \\
\hline Casp-3 & $\begin{array}{c}0.878 * * \\
(0.007)\end{array}$ & $\begin{array}{c}0.928 * * * \\
(<0.001)\end{array}$ & $\begin{array}{c}0.940 * * \\
(0.005)\end{array}$ & $\begin{array}{l}0.887 * \\
(0.019)\end{array}$ & $\begin{array}{l}0.974 * * * \\
(<0.001)\end{array}$ \\
\hline Casp-3 (19kDa) & $\begin{array}{l}0.612 \\
(0.08)\end{array}$ & $\begin{array}{c}0.664 \\
(0.051)\end{array}$ & $\begin{array}{c}0.421 \\
(0.406)\end{array}$ & $\begin{array}{l}0.863^{*} \\
(0.027)\end{array}$ & $\begin{array}{c}0.737 \\
(0.094)\end{array}$ \\
\hline Casp-3 (17kDa) & $\begin{array}{l}0.683 * \\
(0.043)\end{array}$ & $\begin{array}{l}0.740 * \\
(0.023)\end{array}$ & $\begin{array}{c}0.511 \\
(0.301)\end{array}$ & $\begin{array}{l}0.894 * \\
(0.016)\end{array}$ & $\begin{array}{c}0.804 \\
(0.054)\end{array}$ \\
\hline Akt & $\begin{array}{c}0.409 \\
(0.275)\end{array}$ & $\begin{array}{l}0.899 * * * \\
(<0.001)\end{array}$ & $\begin{array}{c}0.236 \\
(0.653)\end{array}$ & $\begin{array}{c}0.201 \\
(0.703)\end{array}$ & $\begin{array}{c}0.052 \\
(0.923)\end{array}$ \\
\hline p-Akt (Ser473) & $\begin{array}{c}0.560 \\
(0.117)\end{array}$ & $\begin{array}{c}0.336 \\
(0.337)\end{array}$ & $\begin{array}{l}0.852 * \\
(0.031)\end{array}$ & $\begin{array}{c}0.372 \\
(0.467)\end{array}$ & $\begin{array}{c}0.634 \\
(0.176)\end{array}$ \\
\hline
\end{tabular}

The following symbols indicate $(*)$ low correlation $(\mathrm{p}<0.05),(* *)$ moderate correlation $(\mathrm{p}<0.01),(* * *)$ high correlation $(\mathrm{p}<0.001),(\dagger \dagger \dagger)$ respectively. N.T. (not tested).

a marker for nitrosative stress [28]. Similar to that seen in the aging male F344xBN [13], we found that aging in the female heart was associated with increased protein tyrosine nitrosylation (Fig. 2). This latter finding is different from previous work using the female Long-Evans/Wistar rat, which found no change in 3-nitrotyrosine levels with cardiac aging [29]. The reasons for differences between the current study and previous is not entirely clear but may be related to differences in the animal strain used and/or animal age as the Long-Evans/Wistar hybrid rats were only 24-month of age at time of sacrifice as opposed to the 26- and 30-month old animals used in the present study.
Similar to our analysis of nitrosative stress, and like that seen in the aging male F344xBN [13], we also observed that aging was associated with increased 4-HNE reactivity (Fig. 3). It is thought that 4-hydroxynonenal levels are a marker of lipid peroxidation and that increased levels of 4-HNE can result in alterations in lipid signaling and enzyme inactivation [30]. Studies have shown that production of 4-HNE occurs primarily from the mitochondria, and that the mitochondria are the primary target for lipid peroxidation damage [31]. This damage to the mitochondria, if extensive, can lead to alterations in ROS production and the release of cyto- 
chrome-C from the mitochondria. To investigate this possibility we next examined if aging was associated with increased evidence of cellular apoptosis. Similar to that seen in the aging male F344XBN hearts [16], TUNEL staining suggested that possibility of increased DNA fragmentation with aging in the female F344xBN heart (Fig. 4). In an effort to elucidate the mechanism of apoptosis, we next investigated the effects of aging on the expression of proteins involved in apoptotic signaling. It is thought that the mitochondrialmediated apoptotic pathway employs the activation of caspase-3 [32]. Here we demonstrate that caspase-3 cleavage was increased with age in the female F344xBN heart (Fig. 5). In a similar fashion, we also found an increase in Bax expression and the ratio of Bax/Bcl-2with aging (Fig. 6). Taken together, and like that previously proposed in the aging male F344xBN heart, these data suggest that age-associated increases in cellular apoptosis are mediated, at least in part, by the mitochondrial pathway.

To examine the degree of relationship between the different dependent variables we calculated the correlation between age, heart weight, number of TUNEL positive cardiomyocytes, HE levels, nitrotyrosine, and 4-HNE. Very high correlation was observed between aging as well as superoxide production, levels of nitrosative-stress marker, and nitrotyrosine (Table 2). Although the exact mechanism(s) cannot be concluded from this data, these findings are consistent with the possibility that the age-associated increases in superoxide production may lead to increased oxidativenitrosative damage that include elevations in the amount of proteins undergoing 4-HNE and nitrotyrosine modification. Interestingly, we also found that age-associated increases in oxidative-nitrosative damage were highly correlated with activation of the mitochondrial-mediated pathway of apoptosis (Table 2). Whether increases in age-related oxidative damage, as suggested by our nitrotyrosine and 4-HNE data,are responsible for initiating cellular apoptosis is currently unclear and beyond the scope of the present study. Nonetheless, the data of the present study demonstrate that aging in the female F344xBN heart is characterized by increased levels of oxidative stress and pro-apoptotic signaling. Whether these biochemical changes result in adaptations in cardiac function is currently unclear and is the focus of ongoing experimentation.

\section{ACKNOWLEDGMENTS}

This study was supported by Department of Energy Grant DE-PS02-09ER09-01 to E.R.B.

\section{REFERENCES}

[1] Phaneuf S, Leeuwenburgh C. Cytochrome c release from mitochondria in the aging heart: a possible mechanism for apoptosis with age. Am J Physiol Regul Integr Comp Physiol 2002; 282(2): R423-30.

[2] Anversa P, Hiler B, Ricci R, Guideri G, Olivetti G. Myocyte cell loss and myocyte hypertrophy in the aging rat heart. J Am Coll Cardiol 1986; 8(6): 1441-8.

[3] Anversa P, Palackal T, Sonnenblick EH, et al. Myocyte cell loss and myocyte cellular hyperplasia in the hypertrophied aging rat heart. Circ Res 1990; 67(4): 871-85.

[4] Olivetti G, Giordano G, Corradi D, et al. Gender differences and aging: effects on the human heart. J Am Coll Cardiol 1995; 26(4): 1068-79.
[5] Olivetti G, Melissari M, Capasso JM, Anversa P. Cardiomyopathy of the aging human heart. Myocyte loss and reactive cellular hypertrophy. Circ Res 1991; 68(6): 1560-8.

[6] Klein M, Kortan C, Kells DI, Dorrington KJ. Equilibrium and kinetic aspects of the interaction of isolated variable and constant domains of light chain with the Fd' fragment of immunoglobulin G. Biochemistry 1979; 18(8): 1473-81.

[7] Jousilahti P, Vartiainen E, Tuomilehto J, Puska P. Sex, age, cardiovascular risk factors, and coronary heart disease: a prospective follow-up study of 14786 middle-aged men and women in Finland. Circulation 1999; 99(9): 1165-72.

[8] Kafonek SD. Postmenopausal hormone replacement therapy and cardiovascular risk reduction. A review. Drugs 1994; 47 Suppl 2: 16-24.

[9] Patten RD, Pourati I, Aronovitz MJ, et al. 17beta-estradiol reduces cardiomyocyte apoptosis in vivo and in vitro via activation of phospho-inositide-3 kinase/Akt signaling. Circ Res 2004; 95(7): 692-9.

[10] Doublier S, Lupia E, Catanuto P, et al. Testosterone and 17betaestradiol have opposite effects on podocyte apoptosis that precedes glomerulosclerosis in female estrogen receptor knockout mice. Kidney Int 2011; 79(4): 404-13.

[11] Lee SD, Kuo WW, Ho YJ, et al. Cardiac Fas-dependent and mitochondria-dependent apoptosis in ovariectomized rats. Maturitas 2008; 61(3): 268-77.

[12] Barp J, Araujo AS, Fernandes TR, et al. Myocardial antioxidant and oxidative stress changes due to sex hormones. Braz J Med Biol Res 2002; 35(9): 1075-81.

[13] Asano S, Rice KM, Kakarla S, et al. Aging influences multiple indices of oxidative stress in the heart of the Fischer 344/NNia x Brown Norway/BiNia rat. Redox Rep 2007; 12(4): 167-80.

[14] Kaprielian RR,Severs NJ. Dystrophin and the cardiomyocyte membrane cytoskeleton in the healthy and failing heart. Heart Fail Rev 2000; 5(3): 221-38.

[15] Rice KM, Blough ER. Sarcopenia-related apoptosis is regulated differently in fast- and slow-twitch muscles of the aging F344/N x BN rat model. Mech Ageing Dev 2006; 127(8): 670-9.

[16] Kakarla SK, Rice KM, Katta A, et al. Possible molecular mechanisms underlying age-related cardiomyocyte apoptosis in the F344XBN rat heart. J Gerontol A Biol Sci Med Sci 2010; 65(2): 147-55.

[17] Arvapalli RK, Paturi S, Laurino JP, et al. Deferasirox decreases age-associated iron accumulation in the aging F344XBN rat heart and liver. Cardiovasc Toxicol 2010; 10(2): 108-16.

[18] Kakarla SK, Fannin JC, Keshavarzian S, et al. Chronic acetaminophen attenuates age-associated increases in cardiac ROS and apoptosis in the Fischer Brown Norway rat. Basic Res Cardiol 2010; 105(4): 535-44.

[19] Wang Y, Wu M, Al-Rousan R, et al. Iron-induced cardiac damage: role of apoptosis and deferasirox intervention. J Pharmacol Exp Ther 2011; 336(1): 56-63.

[20] Turturro A, Witt WW, Lewis S, et al. Growth curves and survival characteristics of the animals used in the Biomarkers of Aging Program. J Gerontol A Biol Sci Med Sci 1999; 54(11): B492-501.

[21] Lipman RD, Chrisp CE, Hazzard DG,Bronson RT. Pathologic characterization of brown Norway, brown Norway x Fischer 344, and Fischer $344 \mathrm{x}$ brown Norway rats with relation to age. J Gerontol A Biol Sci Med Sci 1996; 51(1): B54-9.

[22] Misra MK, Sarwat M, Bhakuni P, Tuteja R,Tuteja N. Oxidative stress and ischemic myocardial syndromes. Med Sci Monit 2009; 15(10): RA209-19.

[23] Pompeia C, Cury-Boaventura MF,Curi R. Arachidonic acid triggers an oxidative burst in leukocytes. Braz J Med Biol Res 2003; 36(11): 1549-60.

[24] Leanderson P, Soderkvist P, Tagesson C,Axelson O. Formation of DNA adduct 8-hydroxy-2'-deoxyguanosine induced by man-made mineral fibres. IARC Sci Publ 1988: 422 - 24.

[25] Ghanizadeh A, Akhondzadeh S, Hormozi M, et al. Glutathionerelated factors and oxidative stress in autism, a review. Curr Med Chem 2012; 19(23): 4000-5.

[26] Judge S, Jang YM, Smith A, Hagen T,Leeuwenburgh C. Ageassociated increases in oxidative stress and antioxidant enzyme activities in cardiac interfibrillar mitochondria: implications for the mitochondrial theory of aging. FASEB J 2005; 19(3): 419-21.

[27] Ramesh T, Kim SW, Sung JH, et al. Effect of fermented Panax ginseng extract (GINST) on oxidative stress and antioxidant 
activities in major organs of aged rats. Exp Gerontol 2012; 47(1): 77-84.

[28] Escobales N,Crespo MJ. Oxidative-nitrosative stress in hypertension. Curr Vasc Pharmacol 2005; 3(3): 231-46.

[29] Leeuwenburgh C, Hansen P, Shaish A, Holloszy JO, Heinecke JW. Markers of protein oxidation by hydroxyl radical and reactive nitrogen species in tissues of aging rats. Am J Physiol 1998; 274(2 Pt 2): R453-61.
[30] McLain AL, Szweda PA, Szweda LI. alpha-Ketoglutarate dehydrogenase: a mitochondrial redox sensor. Free Radic Res 2011; 45(1): 29-36.

[31] Anderson EJ, Katunga LA,Willis MS. Mitochondria as a source and target of lipid peroxidation products in healthy and diseased heart. Clin Exp Pharmacol Physiol 2012; 39(2): 179-93.

[32] Smith MA, Schnellmann RG. Calpains, mitochondria, and apoptosis. Cardiovasc Res 2012; 96(1): 32-7.

Received: September 20, 2013

Revised: October 20, 2013

Accepted: October 22, 2013

(C) Fannin et al.; Licensee Bentham Open.

This is an open access article licensed under the terms of the Creative Commons Attribution Non-Commercial License (http://creativecommons.org/licenses/ by-nc/3.0/) which permits unrestricted, non-commercial use, distribution and reproduction in any medium, provided the work is properly cited. 